\title{
Auditory saccade impairment after central thalamus lesions
}

\author{
M Versino, G Beltrami, C Uggetti, V Cosi
}

\begin{abstract}
Visual and auditory saccades were studied in three patients with an isolated lesion located in the central thalamus. Visual saccades proved to be normal, whereas for auditory stimuli, the amplitude of the first saccade was asymmetric: saccades ipsilateral to the lesion were significantly smaller than those directed to the contralateral side. The patients were able to make a corrective saccade and hence to improve gain and to decrease gain asymmetry. It is suggested that patients were able to localise auditory targets correctly, but did not correctly take into account eye position during the saccade, probably as a consequence of an inaccurate efference copy (corollary discharge) signal. The findings are in keeping with the hypothesis that the central thalamus deals with saccades that are based on extraretinal signals.

(f Neurol Neurosurg Psychiatry 2000;68:234-237)
\end{abstract}

Keywords: saccade, central thalamus, efference copy, magnetic resonance imaging

Dipartimento Scienze

Neurologiche,

Università di Pavia,

Italy

M Versino

$\mathrm{V}$ Cosi

Fondazione IRCCS

Istituto Neurologico C

Mondino, Pavia, Italy

$M$ Versino

C Uggetti

V Cosi

Dipartimento di

Informatica e

Sistemistica,

Università di Pavia,

Italy

G Beltrami

Correspondence to:

Dr Maurizio Versino,

Dipartimento Scienze

Neurologiche, Università di

Pavia, Fondazione IRCCS

Istituto Neurologico C

Mondino, Via Palestro 3,

27100 Pavia, Italy

email mversino@unipv.it

Received 20 May 1999 and in revised form

9 August 1999

Accepted 17 September

1999 bility when standing upright. Her medical history was positive for tobacco smoking and oral contraceptives. Symptom duration was 48 hours. A cerebral CT performed within 24 hours proved to be normal. About 30 days later, neurological examination at our centre showed a slight exacerbation of tendon reflexes in her left arm. Audiometry, brainstem auditory, and somatosensory evoked potentials, automated perimetry, and subjective visual vertical were all normal.

Brain MRI (figure A and B) showed a small ischaemic lesion located in her right thalamus.

PATIENT 2

A 37 year old overweight woman suddenly experienced a complete left ptosis and diplopia in all gaze positions. Neurological examination shortly after symptom onset disclosed complete left (extrinsic+intrinsic) oculomotor paresis, and a tendency to fall to the right. Cerebral CT performed within the first 24 hours was normal, whereas MRI performed the day after showed an ischaemic lesion located in her left thalamus. Referred to our centre 45 days after the onset of symptoms, the patient still showed left ptosis, a left pupil that was larger but reactive to both light and convergence, and a left hypotropia and exotropia due to left superior and medial recti paresis. Audiometry, brainstem auditory and somatosensory evoked potentials, and automated perimetry were all normal. Given the diplopia, the subjective visual vertical was tested in monocular vision alone, and was normal with the left, but not with right, eye viewing; similar findings have been described for oculomotor paresis but not for otolith dysfunction. ${ }^{4}$ Subsequent MRI (figure $\mathrm{C}$ and $\mathrm{D}$ ) confirmed the thalamic lesion.

PATIENT 3

A 30 year old man suddenly experienced vertical diplopia when looking down; it lasted 3 hours. Neurological examination shortly after the resolution of symptoms only showed a slight left superior oblique deficit at Hess screen test. Referred to our centre 14 days after symptom onset, neurological examination, audiometry, brainstem auditory and somatosensory evoked potentials and automated perimetry were all normal. Brain MRI showed an ischaemic lesion located in his left thalamus (figure $\mathrm{E}$ and $\mathrm{F}$ ). 

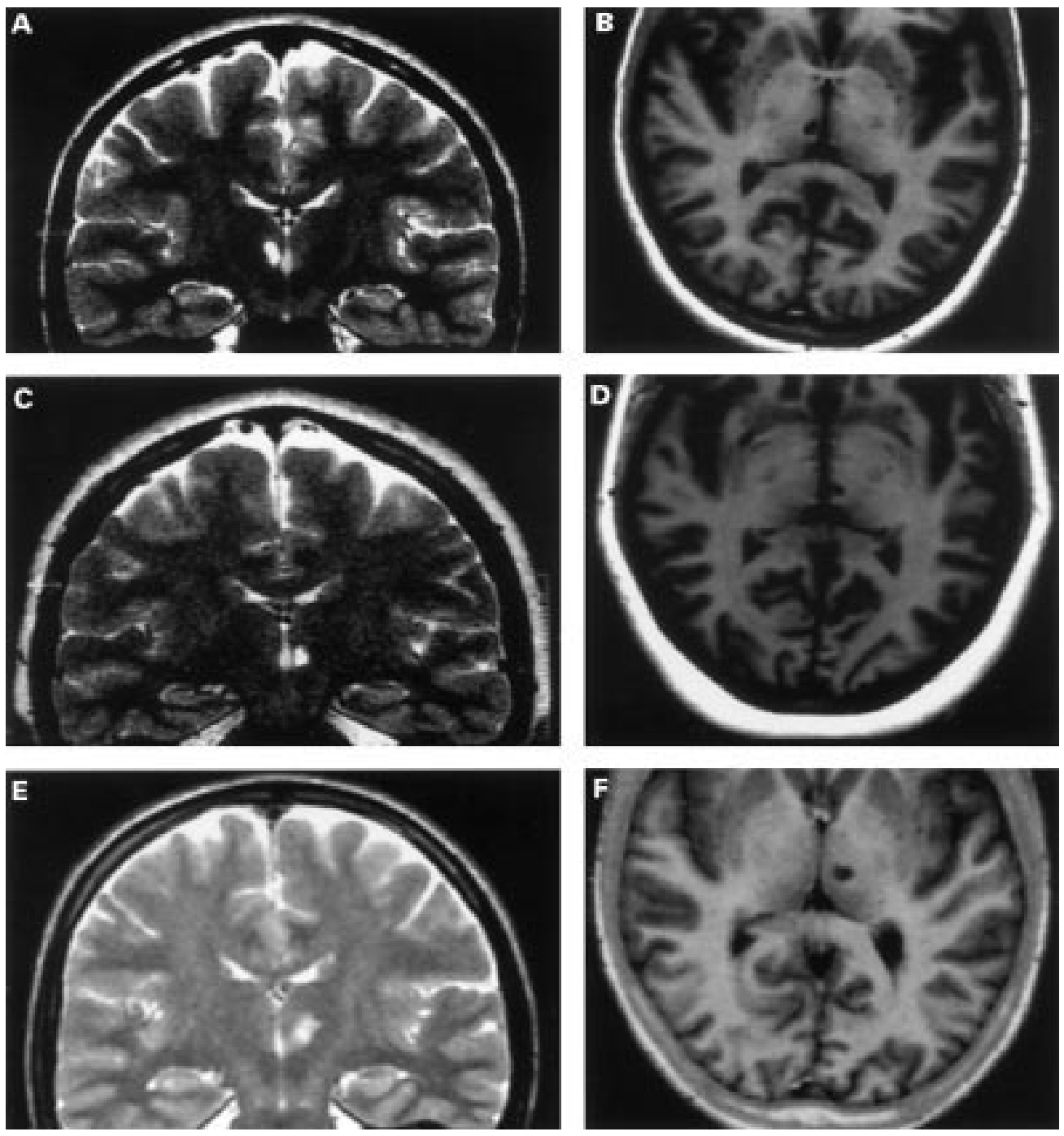

Brain MRI from patients 1 ( $A$ and $B$ ), 2 ( $C$ and $D)$, and 3 ( $E$ and $F$ ). ( $A$ ) spin echo T2 weighted coronal scan $(T R=3000, T E=120)$ shows the product of a lacunar ischaemic lesion-namely, a small, bright, hyperintense area in the parasagittal and anterior portion of the right thalamus; (B) the same lesion is hypointense in inversion recovery $T 1$ weighted axial scan $(T R=3000, T I=600, T E=20) ;(C)$ and $(D)$, and $(E)$ and $(F)$ are the corresponding images for patients 2 and 3, and show a similar lesion in the same position, but in the left thalamus. These lesions probably involved the paraventricular, paratenial, and anteromedial thalamic nuclei.

EYE MOVEMENT RECORDING

The three patients and 10 controls (four men, six women; mean age:29.5 years; range 20-46 years) gave their informed consent for eye movement recording with the magnetic search coil technique (Skalar 3010 system). The recording was performed monocularly from the right eye, in which visual acuity was best in all patients, and was the non-paretic eye in patients 2 and 3 . The coil signal was filtered (band pass $0-75 \mathrm{~Hz}$ ), sampled at $250 \mathrm{~Hz}$, and stored for off line analysis.

The targets were light emitting diodes (LEDs) and loudspeakers positioned at a distance of $80 \mathrm{~cm}$ at 0 , and 10 and 20 degrees left and right to the subject's midsagittal plane. The LED in primary position was lit for 2 seconds and then switched off as a target in eccentric position was activated. In the visually guided saccade task, the LED was presented for 1 second, whereas in the auditory guided saccade task, after 1 second of loudspeaker activation, the corresponding LED was activated for 1 further second to enable the subject to correct for any residual position error using a visually guided saccade. The LED in the primary position was then reilluminated to elicit a recentring saccade. Each peripheral target was activated 12 times in a pseudorandom sequence.

EYE MOVEMENT ANALYSES

For each subject, we measured the latency, duration, peak velocity, and amplitude of each primary centrifugal saccade, and, for auditory saccades alone, we also measured the amplitude of all corrective saccades made during auditory target presentation. For each task and for each direction we computed:

- The duration of a 15 degree saccade derived from the amplitude-duration relationship (linear regression: duration $=\mathrm{a}+\mathrm{b} \times$ saccade amplitude)

- The maximal peak velocity value for a theoretical saccade amplitude equal to infinite derived from the amplitude peak velocity relation (peak velocity $=$ saccade amplitude/ $(c+d \times$ saccade amplitude $))$ 
Table 1 Data from the three patients and from the control group

\begin{tabular}{|c|c|c|c|c|c|c|}
\hline & \multicolumn{3}{|c|}{ Patients } & \multicolumn{3}{|l|}{ Controls } \\
\hline & 1 & 2 & 3 & Mean (SD) & $M e a n \pm 2 S D$ & Observed range \\
\hline G right visual & 0.93 & 1.00 & 0.89 & $0.919(0.044)$ & $0.83: 1.01$ & 0.84 to 0.99 \\
\hline G left visual & 0.94 & 0.94 & 0.92 & $0.915(0.047)$ & $0.82: 1.01$ & 0.86 to 0.99 \\
\hline G asymmetry visual & 0.01 & -0.07 & 0.03 & $-0.004(0.046)$ & $-0.10: 0.09$ & -0.07 to 0.07 \\
\hline G right auditory & 0.85 & 0.8 & 0.74 & $0.907(0.194)$ & $0.52: 1.29$ & 0.69 to 1.22 \\
\hline G left auditory & 1.06 & 0.59 & 0.61 & $0.955(0.202)$ & $0.55: 1.36$ & 0.75 to 1.33 \\
\hline $\mathrm{G}$ asymmetry auditory & 0.22 & -0.3 & -0.19 & $0.052(0.068)$ & $-0.08: 0.19$ & -0.07 to 0.12 \\
\hline G asymmetry vis-aud & -0.21 & 0.23 & 0.22 & $-0.057(0.066)$ & $-0.19: 0.07$ & -0.16 to 0.06 \\
\hline Final G right & 0.94 & 0.92 & 0.84 & $0.952(0.097)$ & $0.76: 1.14$ & 0.82 to 1.16 \\
\hline Final G left & 1.00 & 0.99 & 0.89 & $0.982(0.064)$ & $0.85: 1.1$ & 0.88 to 1.1 \\
\hline Final G asymmetry & 0.062 & 0.073 & 0.069 & $0.033(0.073)$ & $-0.11: 0.18$ & -0.11 to 0.12 \\
\hline
\end{tabular}

For controls, we report the mean and the standard deviation (SD) values, the normal range computed as the mean $\pm 2 \mathrm{SD}$, and the observed range. Abnormal values are reported in bold. $\mathrm{G}=$ mean gain, where gain is the ratio of first saccade amplitude to target displacement. Final gain is the ratio of the amplitude of all saccades made during auditory target presentation to target displacement. Right=rightward saccades; left=leftward saccades; visual=visual saccade task; auditory=auditory saccade task; asymmetry=the difference between leftward and rightward saccade values of a given variable divided by the mean of these values; vis-aud=the difference between visual and auditory values.

- The mean gain value, where individual gain values correspond to the ratio of first saccade amplitude to target displacement

- For auditory saccades alone the mean final gain value was measured, where final gain was computed by adding the amplitude of all corrective saccades made during auditory target presentation to that of first saccade, and by considering the ratio of this global amplitude to target displacement

- The mean latency value.

Moreover, for each subject and for each variable, we computed an asymmetry index to quantify the difference between leftward and rightward saccades, account being taken for the mean value of the variable in that subject. For instance, the asymmetry index for gain is:

$$
\text { Gasymmetry }=\frac{\text { Gleft-Gright }}{\frac{\text { Gleft+Gright }}{2}}
$$

Finally, for each variable and for each asymmetry index we computed the difference between visual and auditory tasks.

For each variable, we defined the normal range as the mean $\pm 2 \mathrm{SD}$ provided that the hypothesised normal distribution of that variable was not rejected by the KolmogorovSmirnov test (the significance level was set as $\mathrm{p}=0.05)$.

Moreover, for each variable we compared the mean value of the two groups by the MannWhitney test; in these mean value comparisons both the asymmetry indices and the differences between visual and auditory task were considered as absolute values.

\section{Results}

We considered all the variables to be normally distributed on the basis of KolmogorovSmirnov tests for normal distribution, which were always non-significant.

In the visual task, all variables, including asymmetry indices, were normal and there were no differences between patient and control mean values. The table shows the data for visual saccade gain.

In the auditory task, duration, peak velocity, and latency and the corresponding asymmetry indices were normal in all patients. However, whereas all patients again showed normal gain in both directions, ipsilateral saccades were significantly smaller than those contralateral to the lesion side. The gain asymmetry index was larger than both computed normal limits and observed range in controls (table 1). In absolute value, the patients' smallest gain asymmetry index was $157 \%$ greater than the controls' largest value.

In the auditory task, the only significant difference detectable by mean value comparisons concerned the gain asymmetry (absolute values), which was larger in patients than in controls (0.77 $v 0.23$; Mann-Whitney test: $z=0.53, \mathrm{p}=0.011)$.

In all patients, all differences between visual and auditory variables were normal with the exception of gain asymmetry, which was larger than both the computed normal range and observed range in controls. Again, in absolute values the patients' smallest asymmetry index was $129 \%$ greater than the controls' largest value.

For the differences between corresponding visual and auditory variables, the only significant difference detectable by mean value comparisons was for gain asymmetry (absolute values), which was larger in patients than in controls (0.70 $v 0.22$; Mann-Whitney test: $z=2.53, \mathrm{p}=0.011$ ).

All patients were able to make corrective saccades and hence to improve their accuracy and reduce the asymmetry index. All the final gain values fell within the normal limits computed in controls (table), and there were no significant differences detectable by mean value comparisons.

\section{Discussion}

Our results show some abnormalities detectable in the auditory but not in the visual task. Although the occurrence of diplopia suggests midbrain involvement, ${ }^{5}$ the isolated lesions in the central thalamus were the only abnormalities detectable by otherwise normal MRI. Moreover, visual saccade proved to be normal, a finding which argues against the possible involvement of brainstem or cerebellar structures, which generate and determine the accuracy of horizontal saccades.

The gain asymmetry detected in the auditory paradigm might derive from an incorrect 
localisation of target position. However, MRI did not show any lesion along the auditory pathways, the auditory function was normal in all patients, and latencies for auditory saccades were normal and symmetric. Additionally, during presentation of an auditory target, all patients made corrective saccades that significantly improved gain and decreased gain asymmetry. Overall, we consider a dysfunction in locating auditory targets an unlikely explanation for the gain asymmetry.

An alternative hypothesis is that the localisation of eye position was possibly faulty. This faulty localisation was probably a result of inadequate monitoring of eye position during, but not after, the end of the saccade. Again, all patients were able to make successful corrective saccades, which implies that after a saccade they were able to check both target and eye positions, and hence to evaluate the residual position error.

The efference copy signal is an internal copy of motor command which is fed back to update the ocular motor structures that provide saccade programming about eye position. ${ }^{6}$

According to anatomical data, ${ }^{1}$ the central thalamus receives projections from brainstem premotor structures and projects to the ocular motor frontal and parietal cortex, which are connected to the superior colliculus, and from there, back again to the brain stem premotor and motor structures. Moreover, the central thalamus contains cells that code eye position, ${ }^{7}$ either with contraversive or with ipsiversive preference. ${ }^{1}$ Accordingly, the central thalamus is a likely candidate to receive and to project an efference copy signal.

In two patients with isolated thalamic lesions reported by Gaymard et $a l{ }^{3}$ memory guided saccades were accurate when the position error could be memorised in retinal coordinates, but not when an extraretinal position error (eye displacement) had to be taken into account.
This finding suggested that the efferent copy for extraretinal saccade was not calibrated or that it was not correctly distributed to cortical ocular motor centres.

In our patients, the abnormality was more subtle (auditory saccades were not dysmetric), and suggested a saccade direction dependent bias in the efferent copy signal, although this bias could be compensated for by corrective saccades. We are not able to explain the reason why this bias always resulted in smaller saccades when directed toward the lesioned side than in the opposite direction. Table 1 of Gaymard et $a l^{\beta}$ shows a similar finding in all paradigms including the retinotopic memory guided paradigm. However, the authors did not comment on this finding, and from the data available to the reader it is not possible to establish whether the patients' gain asymmetry was significantly larger than in controls.

In conclusion, our three patients with an isolated thalamic lesion all showed the same abnormality in auditory but not in visual saccade, which is consistent with the hypothesis that the central thalamus is involved in extraretinal saccade control.

We are grateful to David S Zee for his helpful suggestions.

1 Schlag-Rey M, Schlag J. The central thalamus. In: Wurtz $\mathrm{RH}$, Goldberg ME, eds. The neurobiology of saccadic system. Amsterdam: Elsevier, 1989:361-90.

2 Cramon von D, Zihl J. Roving eye movements with bilateral symmetrical lesions of the thalamus. F Neurol 1979;221: 105-12.

3 Gaymard B, Rivaud S, Pierrot-Deseilligny C. Impairment of extraretinal eye position signals after central thalamic lesions in humans. Exp Brain Res 1994;192:1-9.

4 Dieterich M, Brandt T. Ocular torsion and perceived vertical in oculomotor, trochlear and abducens nerve palsies. Brain 1993;116:1095-104.

5 Siatkowski RM, Schatz NJ, Sellitti TP, et al. Do thalamic lesions really cause vertical gaze palsies? f Clin Neurolesions really cause vertical
ophthalmol 1993;13:190-3.

6 Leigh RJ, Zee DS. The neurology of eye movements. Philadelphia: FA Davis, 1991

7 Schlag-Rey M, Schlag J. Visuomotor functions of central thalamus in monkey. 1: Unit activity related to spontaneous eye movements. F Neurophysiol 1984;51:1149-73. 